\title{
Determining the optimal strategies to achieve elimination of transmission for Schistosoma mansoni
}

Klodeta Kura ${ }^{1,2,3^{*}}$, Diepreye Ayabina ${ }^{4}$, T. Deirdre Hollingsworth ${ }^{4}$ and Roy M. Anderson ${ }^{1,2,3,5}$

\begin{abstract}
Background: In January 2021, the World Health Organization published the 2021-2030 roadmap for the control of neglected tropical diseases (NTDs). The goal for schistosomiasis is to achieve elimination as a public health problem (EPHP) and elimination of transmission (EOT) in 78 and 25 countries (by 2030), respectively. Mass drug administration (MDA) of praziquantel continues to be the main strategy for control and elimination. However, as there is limited availability of praziquantel, it is important to determine what volume of treatments are required, who should be targeted and how frequently treatment must be administered to eliminate either transmission or morbidity caused by infection in different endemic settings with varied transmission intensities.

Methods and Results: In this paper, we employ two individual-based stochastic models of schistosomiasis transmission developed independently by the Imperial College London (ICL) and University of Oxford (SCHISTOX) to determine the optimal treatment strategies to achieve EOT. We find that treating school-age children (SAC) only is not sufficient to achieve EOT within a feasible time frame, regardless of the transmission setting and observed age-intensity of infection profile. Both models show that community-wide treatment is necessary to interrupt transmission in all endemic settings with low, medium and high pristine transmission intensities.
\end{abstract}

Conclusions: The required MDA coverage level to achieve either transmission or morbidity elimination depends on the prevalence prior to the start of treatment and the burden of infection in adults. The higher the worm burden in adults, the higher the coverage levels required for this age category through community-wide treatment programmes. Therefore, it is important that intensity and prevalence data are collected in each age category, particularly from SAC and adults, so that the correct coverage level can be calculated and administered.

Keywords: Schistosomiasis, Mathematical models, Individual-based stochastic models, Mass drug administration, School-based treatment, Community-wide treatment, Elimination of transmission, Elimination as a public health problem

\section{Background}

Schistosomiasis is an intestinal or urogenital disease caused by infection with Schistosoma trematode species, predominantly Schistosoma mansoni, S. japonicum and $S$. haematobium [1]. An individual becomes infected when

\footnotetext{
*Correspondence: k.kura@imperial.ac.uk

${ }^{1}$ London Centre for Neglected Tropical Disease Research, London, UK

Full list of author information is available at the end of the article
}

the cercarial larval forms of the parasite (released by the intermediate host, which are various species of freshwater snails) penetrate the skin during contact with contaminated water. Symptoms of intestinal schistosomiasis can vary from abdominal pain, blood in stool, diarrhoea and Katayama fever, to enlargement of the liver and spleen, fibrosis, portal hypertension and accumulation of fluid in the peritoneal cavity in most advanced stages. Urogenital original author(s) and the source, provide a link to the Creative Commons licence, and indicate if changes were made. The images or other third party material in this article are included in the article's Creative Commons licence, unless indicated otherwise in a credit line to the material. If material is not included in the article's Creative Commons licence and your intended use is not permitted by statutory regulation or exceeds the permitted use, you will need to obtain permission directly from the copyright holder. To view a copy of this licence, visit http://creativecommons.org/licenses/by/4.0/. The Creative Commons Public Domain Dedication waiver (http://creativeco mmons.org/publicdomain/zero/1.0/) applies to the data made available in this article, unless otherwise stated in a credit line to the data. 
schistosomiasis can cause bloody urine, kidney damage and bladder cancer in the most severe cases $[2,3]$.

Mass drug administration (MDA), using the drug praziquantel (PZQ), is the main form of control at present, alongside behaviour modification and improvement in sanitation. Current work on potential vaccine candidates is promising, but at an early stage at present [4-7].

According to the World Health Organization (WHO), an estimated 236 million people require MDA worldwide, of which 90\% live in Africa [8]. Schistosomiasis is endemic in 78 countries, of which 51 countries have moderate to high prevalence. In 2016 nearly 24,000 people died from schistosomiasis, although in reality this number is believed to be higher, as very rarely is schistosomiasis recorded in death certificates.

Morbidity control, increasing treatment coverage to at least $75 \%$ in all school-age children (SAC; $5-14$ years of age), and elimination of transmission (EOT) in the Americas and Western Pacific regions and some parts of the African Region were the targets set in the 2020 World Health Organization (WHO) roadmap [9]. To achieve these targets, WHO recommended treatment guidelines based on the baseline prevalence of infection in SAC [10]. These guidelines focused on treating SAC, as they are more likely to be infected, but inclusion of adults ( $\geq 15$ years old) at risk of infection through occupation and/or living in high-transmission areas has also been recommended.
The fifth progress report assessing progress on the goals set in the London Declaration has scored schistosomiasis red in 2016 as there was no significant measurable progress towards the elimination target [11].

However, there has been good progress since 2016 in SAC MDA coverage (Fig. 1), partly due to the donation of 250 million PZQ tablets annually by Merck \& Co. through WHO, primarily for SAC [8]. There has been low coverage in adults over the years, with figures not exceeding $20 \%$ coverage for any given country (Fig. 1). As previous research has shown, high coverage of adults is needed in many moderate- to high-transmission settings to achieve elimination as a public health problem (EPHP) $[6,12-14]$.

In January 2021, WHO issued the 2021-2030 roadmap for neglected tropical diseases (NTDs) [8]. The goal for schistosomiasis is EPHP, to be reached by 2023 in 49 countries, by 2025 in 69 countries and by 2030 in 78 countries (Table 1). This is to be achieved by reducing the heavy-intensity (eggs per gram $\geq 400$ ) prevalence in SAC to less than $1 \%$.

The end goal for schistosomiasis is interruption of transmission, achieved when the incidence of new infections reduces to zero. The WHO roadmap suggests this should be reached by 2030 in 25 countries.

In previous work [13], we have explored the strategies in the design of MDA programmes needed to be adopted to achieve and sustain the elimination of schistosomiasis

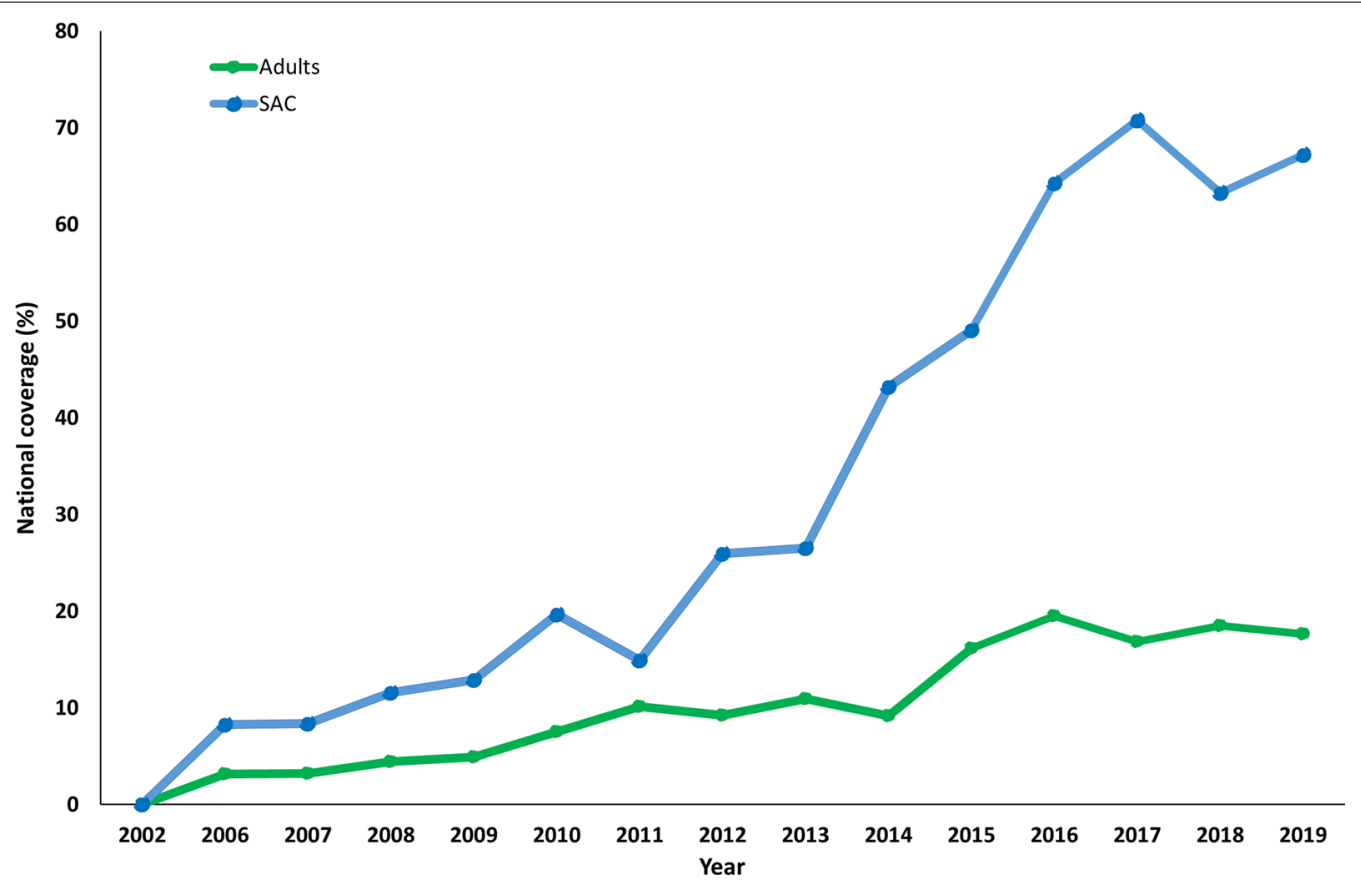

Fig. 1 National coverage (for SAC and adults) in endemic regions as reported by the World Health Organization (WHO) preventive chemotherapy and transmission control (PCT) databank [35] 
Table 1 WHO 2030 targets, sub-targets and milestones for EPHP, achieved when the heavy-intensity prevalence in SAC reduces to less than $1 \%[8]$

\begin{tabular}{llll}
\hline Indicator & 2023 & 2025 & 2030 \\
\hline $\begin{array}{l}\text { Number of countries validated for EPHP } \\
\text { Number of countries where absence of infection in humans }\end{array}$ & $49 / 78(63)$ & $69 / 78(88 \%)$ & $78 / 78(100 \%)$ \\
has been achieved & $10 / 78(13 \%)$ & $19 / 78(24 \%)$ & $25 / 78(32 \%)$
\end{tabular}

as a public health problem. This past work has focused on the MDA coverage required across all child and adult age groups to achieve specified targets by a defined year.

In this present paper, we analyse whether it is possible to achieve the interruption of transmission by exploring various treatment strategies.

\section{Methods}

In this paper, we use two individual-based stochastic models developed independently by Imperial College London (ICL) and the University of Oxford (SCHISTOX). Details of the two transmission dynamics models have been published previously [15-19]. Both models have similar processes, except for one important difference. The ICL model assumes that the number of eggs produced is a non-linear function (density-dependent egg production) of the female worm burden assuming monogamous sexual reproduction. In contrast, SCHISTOX assumes that the number of eggs produced is proportional to the number of worm pairs (male and female worms). Both models consider a single shared environmental reservoir of infection and collapse the details of the dynamics of the snail intermediate host and the two larval stages into the expression for the basic reproduction number, $R_{0}$, due to the huge differences between the lifespan of the different life-cycle stages (hours for miracidia and cercaria, days to weeks for infected snails, and 3.5-10.5 years for the adult parasite in humans). The two models have been calibrated to produce the same baseline settings by varying the basic reproduction number, $\mathrm{R}_{0}$, in the ICL model, and the overall contact rate in the SCHISTOX model. Existing estimates for the basic reproduction number for schistosomiasis are typically in the range of 1-4 [15-19]. In our simulations, to analyse the impact of MDA in reducing the prevalence of disease, we assume a population of 500 individuals without migration and random coverage at each round of treatment, e.g., at each MDA round a new random defined fraction of the target group is treated. Persistent noncompliance is not addressed hence the predictions may be on the optimistic side. We also consider two ageintensity profiles of infection, corresponding to low and high adult burden of infection, achieved by varying the age-specific contact rates (Table 2). A description of all parameters used, and their values are given in Table 2.

\section{Scenarios simulated}

In our simulations, we consider low (SAC prevalence $<10 \%$ ), moderate (SAC prevalence $10-50 \%$ ) and high (SAC prevalence $\geq 50 \%$ ) baseline prevalence settings, with a low or high adult burden of infection (Table 1). These reflect different pristine transmission settings as defined by the magnitude of the basic reproductive number, $\mathrm{R}_{0}$.

Previous mathematical work [13] has shown that it is possible to achieve and sustain the EPHP goal by adopting various MDA treatment strategies. The probability of achieving and maintaining this goal depends

Table 2 Parameter values used for S. mansoni (for both ICL and SCHISTOX model)

\begin{tabular}{|c|c|c|}
\hline Parameter & Value & References \\
\hline Fecundity & 0.34 eggs/female/sample & {$[1,36,37]$} \\
\hline Aggregation parameter & 0.04 for low-prevalence settings; 0.24 for high-prevalence settings & {$[1,38]$} \\
\hline Density-dependent fecundity & $0.0007 /$ female worm & {$[1,39]$} \\
\hline Worm lifespan & 5.7 years & {$[1,16,40]$} \\
\hline Drug efficacy & $86.3 \%$ & {$[41]$} \\
\hline $\begin{array}{l}\text { Low adult burden setting: age-specific contact rates } \\
\text { for } 0-4,5-9,10-15,16+\text { years old }\end{array}$ & $0.01,1.2,1,0.02$ & [39] \\
\hline $\begin{array}{l}\text { High adult burden setting: age-specific contact rates } \\
\text { for } 0-4,5-11,12-19,20+\text { years old }\end{array}$ & $0.01,0.61,1,0.12$ & [39] \\
\hline Prevalence of infection & Percentage of population having $>0$ eggs per gram [epg] & - \\
\hline Heavy-intensity infection prevalence & Percentage of population having $\geq 400 \mathrm{epg}$ & [42] \\
\hline
\end{tabular}


on the baseline prevalence prior to treatment, the precise form of the age-intensity profile of infection and the strategy adopted in terms of MDA coverage and treatment frequency.

Building on this past published research, we explore whether we can achieve EOT by considering the following scenarios:

1. Can we move from EPHP to EOT? In this scenario, we explore whether we can move towards EOT after achieving EPHP. We continue the MDA programme (once the EPHP is reached) by giving PZQ annually to $75 \%$ of SAC (the WHO-recommended treatment coverage) only for up to a further 10 years. If the EOT goal has not been met, we increase the SAC coverage or/and include adults in the treatment programme so that the goal can be met within 10 years.

2. Can we achieve EOT within 15 years of the start of the programme (from baseline) by intensifying the treatment strategies with more frequent treatment and higher MDA coverage levels? In this scenario, we do not have the EPHP goal as a sub-target. We consider various MDA coverage combinations in SAC and adults and find whether the EOT target can be achieved.

At the end of the programme, we evaluate the population prevalence (i.e., across all age groups) to determine whether the EOT has been met. We consider the EOT to be achieved when the overall prevalence is reduced to less than $1 \%$ by single Kato-Katz on two samples per individual using a sample size of 200 individuals from all age categories, regardless of the burden of infection in adults [20].
Each scenario is run 500 times and the EOT goal is considered achieved when at least $90 \%$ of simulations are below $1 \%$.

\section{Results}

\section{Can we move from EPHP to EOT?}

Here we present results and analyse what is required to achieve EOT after achieving EPHP. Table 3 presents the results for low adult burden of infection and Table 4 presents results for the example of a high adult burden of infection.

\section{Low-transmission settings}

In low-transmission settings with a low adult burden of infection (Table 3), we find that annual treatment of $75 \%$ of SAC alone can achieve the EPHP goal within 3 years. Continuing MDA with the same coverage for another 5-6 years (depending on the baseline prevalence) can achieve the EOT with high probability. Therefore, up to 9 years is required to achieve the EOT goal from the start of the programme (at baseline).

For high adult burden of infection (Table 4), as expected, it takes longer for these goals to be achieved. The EPHP can be met within 4 years of treatment, while the EOT can be achieved within 9 years after the EPHP has been met. For both these goals, coverage of $75 \%$ of SAC has been used. Thus, in total it takes up to 13 years for the EOT goal to be met from the start of the MDA programme with high annual coverage of SAC.

\section{Moderate-transmission settings}

For low adult burden of infection (Table 3), our models predict that treating $75 \%$ of SAC can achieve the EPHP goal within 5 years of treatment. After the EPHP goal has been achieved, we can restart the programme. We find that treating $75 \%$ of SAC only cannot achieve the EOT

Table 3 Model-recommended treatment strategies for achieving EPHP and EOT (after the achievement of EPHP) for low adult burden of infection. Results for low-transmission settings are generated using the ICL model

\begin{tabular}{|c|c|c|c|}
\hline $\begin{array}{l}\text { Prevalence in SAC } \\
\text { prior to treatment }\end{array}$ & Model & $\begin{array}{l}\text { Model-recommended treatment strategy for achieving } \\
\text { EPHP [13] }\end{array}$ & $\begin{array}{l}\text { Model-recommended treatment strategy for achieving } \\
\text { EOT after the achievement of EPHP }\end{array}$ \\
\hline $\operatorname{Low}(<10 \%)$ & $\mathrm{ICL}$ & $75 \%$ SAC for $2-3$ years & $75 \%$ SAC and $30 \%$ adults for $5-6$ years \\
\hline \multirow[t]{2}{*}{ Moderate (10-50\%) } & $\mathrm{ICL}$ & $75 \%$ SAC for 4 years & $75 \%$ SAC and $40 \%$ adults for $8-9$ years \\
\hline & SCHISTOX & $75 \%$ SAC for 5 years & $75 \%$ SAC and $40 \%$ adults for $6-7$ years \\
\hline \multirow[t]{2}{*}{ High ( $\geq 50 \%)$} & $\mathrm{ICL}$ & $\begin{array}{l}75 \% \text { SAC for } 4-10 \text { years (baseline prevalence below } 67 \% \\
\text { SAC }+56 \% \text { adults) } \\
90 \% \text { SAC and } 45 \% \text { adults for } 10 \text { years (baseline preva- } \\
\text { lence: } 71 \% \text { SAC }+60 \% \text { adults) }\end{array}$ & $\begin{array}{l}75 \% \text { SAC and } 50 \% \text { adults for } 8-10 \text { years (baseline preva- } \\
\text { lence below } 67 \% \text { SAC }+56 \% \text { adults) } \\
75 \% \text { SAC and } 50 \% \text { adults for } 5 \text { years (baseline prevalence: } \\
71 \% \text { SAC }+60 \% \text { adults) }\end{array}$ \\
\hline & SCHISTOX & $\begin{array}{l}75 \% \text { SAC for } 5-10 \text { years (baseline prevalence below } 76 \% \\
\text { SAC }+42 \% \text { adults) } \\
90 \% \text { SAC and } 45 \% \text { adults for } 8 \text { years (baseline prevalence: } \\
79 \% \text { SAC }+49 \% \text { adults) }\end{array}$ & $\begin{array}{l}75 \% \text { SAC and } 50 \% \text { adults for } 7-8 \text { years (baseline preva- } \\
\text { lence below } 76 \% \text { SAC }+42 \% \text { adults) } \\
75 \% \text { SAC and } 50 \% \text { adults for } 5 \text { years (baseline prevalence: } \\
79 \% \text { SAC }+49 \% \text { adults) }\end{array}$ \\
\hline
\end{tabular}


Table 4 Model-recommended treatment strategies for achieving EPHP and EOT (after the achievement of EPHP) for high adult burden of infection. Results for low-transmission settings are generated using the ICL model

\begin{tabular}{|c|c|c|c|}
\hline $\begin{array}{l}\text { Prevalence in SAC } \\
\text { prior to treatment }\end{array}$ & Model & $\begin{array}{l}\text { Model-recommended treatment strategy for achieving } \\
\text { EPHP }\end{array}$ & $\begin{array}{l}\text { Model-recommended treatment strategy for achieving } \\
\text { EOT after the achievement of EPHP }\end{array}$ \\
\hline Low $(<10 \%)$ & $\mathrm{ICL}$ & $75 \%$ SAC for $2-4$ years & $75 \%$ SAC and $30 \%$ adults for $8-9$ years \\
\hline \multirow[t]{2}{*}{ Moderate (10-50\%) } & $\mathrm{ICL}$ & $75 \%$ SAC for $5-7$ years & $75 \%$ SAC and $55 \%$ adults for $9-10$ years \\
\hline & SCHISTOX & $75 \%$ SAC for $6-8$ years & $75 \%$ SAC and $55 \%$ adults for 7 years \\
\hline \multirow[t]{2}{*}{ High ( $\geq 50 \%)$} & $\mathrm{ICL}$ & $\begin{array}{l}90 \% \text { SAC and } 45 \% \text { adults for } 5-6 \text { years (baseline preva- } \\
\text { lence below } 60 \% \text { SAC }+63 \% \text { adults) } \\
95 \% \text { SAC and } 85 \% \text { adults for } 10 \text { years (baseline preva- } \\
\text { lence: } 71 \% \text { SAC }+72 \% \text { adults) }\end{array}$ & $\begin{array}{l}75 \% \text { SAC and } 55 \% \text { adults for } 7-8 \text { years (baseline preva- } \\
\text { lence below } 60 \% \text { SAC }+63 \% \text { adults) } \\
85 \% \text { SAC and } 75 \% \text { adults for } 9 \text { years (baseline prevalence: } \\
71 \% \text { SAC }+72 \% \text { adults) }\end{array}$ \\
\hline & SCHISTOX & $\begin{array}{l}\text { 90\% SAC and } 45 \% \text { adults for } 4-8 \text { years (baseline preva- } \\
\text { lence below } 76 \% \text { SAC }+64 \% \text { adults) } \\
\text { 95\% SAC and } 85 \% \text { adults for } 8 \text { years (baseline prevalence: } \\
79 \% \text { SAC }+72 \% \text { adults) }\end{array}$ & $\begin{array}{l}75 \% \text { SAC and } 55 \% \text { adults for } 6-7 \text { years (baseline preva- } \\
\text { lence below } 76 \% \text { SAC }+64 \% \text { adults) } \\
85 \% \text { SAC and } 75 \% \text { adults for } 8 \text { years (baseline prevalence: } \\
79 \% \text { SAC }+72 \% \text { adults) }\end{array}$ \\
\hline
\end{tabular}

goal. However, including adults in the treatment programme can increase the probability of achieving this goal. We find that using coverage of $75 \%$ among SAC and $40 \%$ among adults can achieve the EOT goal within 9 years.

For high adult burden of infection (Table 4), the EPHP is achieved within 8 years by following the WHO-recommended treatment strategy of $75 \%$ SAC. We need to continue the programme (after the achievement of EPHP) for up to 10 more years and use a coverage of at least $75 \%$ SAC and 55\% adults to achieve the EOT goal.

\section{High-transmission settings}

For low adult burden of infection (Table 3 ) and SAC baseline prevalence less than $67 \%$, the ICL model predicts that administering MDA to $75 \%$ of SAC can achieve the EPHP goal within 10 years. An extra 10 years and a coverage of $75 \%$ of SAC and $50 \%$ adults might be needed to achieve EOT. For baseline prevalence above this threshold (67\%), we need to increase the SAC coverage and include adults in the treatment programme to achieve EPHP within 10 years. Administering MDA to $90 \%$ of SAC and $45 \%$ of adults can achieve this goal within 10 years. After this point, another 5 years and a coverage of $75 \%$ among SAC and $50 \%$ among adults are required to achieve the EOT goal.

In contrast, the SCHISTOX model predicts that treating $75 \%$ of SAC can achieve the EPHP goal within 10 years for baseline prevalence less than $76 \%$. An extra 5 years are required to achieve the EOT. For baseline prevalence above this threshold, 8 years are required to achieve EPHP and an extra 5 years to achieve EOT.

For high adult burden of infection (Table 4), the ICL model predicts that EPHP can be achieved by treating $90 \%$ of SAC and $45 \%$ of adults annually for up to 6 years if the baseline SAC prevalence is below 60\%. After achieving EPHP, the EOT can be achieved by treating $75 \%$ of SAC and $55 \%$ of adults annually for up to 8 years. Thus, it takes up to 14 years to achieve the EOT since the start of the MDA programme (at baseline).

The SCHISTOX model, predicts that EPHP can be achieved by treating $90 \%$ of SAC and $45 \%$ of adults annually for up to 8 years if the baseline SAC prevalence is below 76\%. After achieving EPHP, the EOT can be achieved by treating $75 \%$ of SAC and $55 \%$ of adults annually for up to 7 years. Therefore, using the SCHISTOX model, it takes up to 15 years to achieve the EOT target from the start of the MDA programme.

For baseline SAC prevalence above these thresholds, intensified treatment is needed, such as higher coverage of SAC and adults.

\section{Intensive treatment strategies}

Here we do not have the elimination of a public health problem as a sub-target. This strategy can be considered in low-transmission settings where the only goal from the start of the treatment programme is EOT. For example, the ongoing Geshiyaro Project in southern Ethiopia is assessing various treatment strategies to achieve EOT for schistosomiasis and soil-transmitted helminths (STH) within 6 years of treatment [21].

In Fig. 2, we have presented results on the number of years of annual treatment to achieve EOT of $S$. mansoni as a function of coverage of SAC versus adults for lowtransmission settings and low/high worm burden of infection in adults.

These results show that treating SAC only cannot achieve EOT within 15 years of treatment, regardless of the burden of infection in adults. This is due to the fact that the untreated adults continue to contribute to the reservoir of infection, making it impossible to break transmission. Community-wide treatment (SAC and 


\section{Worm burden in adults}

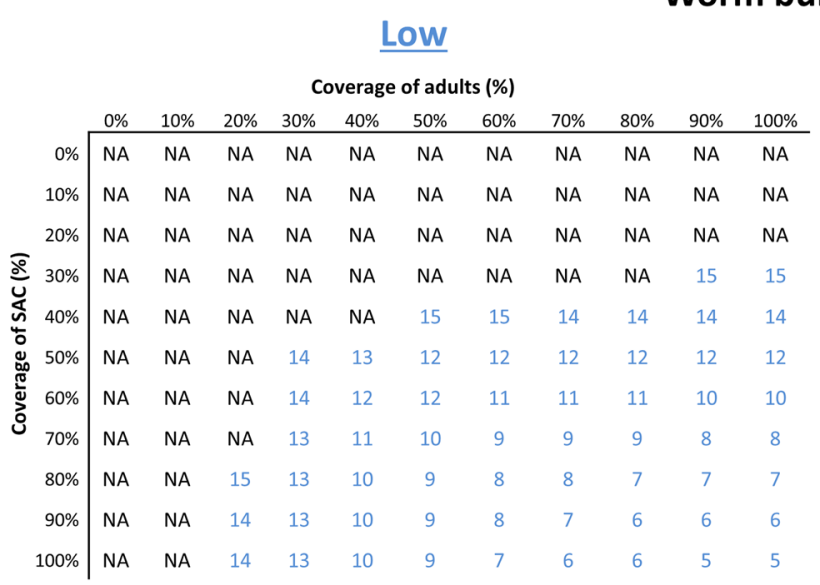

\begin{tabular}{|c|c|c|c|c|c|c|c|c|c|c|c|}
\hline & & & & & Covera & e of & ults (\% & & & & \\
\hline & $0 \%$ & $10 \%$ & $20 \%$ & $30 \%$ & $40 \%$ & $50 \%$ & $60 \%$ & $70 \%$ & $80 \%$ & $90 \%$ & $100 \%$ \\
\hline $0 \%$ & NA & NA & NA & NA & NA & NA & NA & NA & NA & NA & NA \\
\hline $10 \%$ & NA & NA & NA & NA & NA & NA & NA & NA & NA & NA & NA \\
\hline $20 \%$ & NA & NA & NA & NA & NA & NA & NA & NA & NA & NA & NA \\
\hline $30 \%$ & NA & NA & NA & NA & NA & NA & NA & NA & NA & NA & NA \\
\hline $40 \%$ & NA & NA & NA & NA & NA & NA & NA & NA & 15 & 14 & 14 \\
\hline $50 \%$ & NA & NA & NA & NA & NA & NA & 14 & 13 & 13 & 12 & 12 \\
\hline $60 \%$ & NA & NA & NA & NA & NA & 15 & 12 & 12 & 11 & 10 & 10 \\
\hline $70 \%$ & NA & NA & NA & NA & 15 & 13 & 11 & 10 & 9 & 9 & 9 \\
\hline $80 \%$ & NA & NA & NA & NA & 15 & 13 & 11 & 9 & 9 & 8 & 7 \\
\hline $90 \%$ & NA & NA & NA & NA & 15 & 13 & 10 & 9 & 8 & 7 & 7 \\
\hline $100 \%$ & NA & NA & NA & NA & 15 & 12 & 10 & 8 & 7 & 7 & 6 \\
\hline
\end{tabular}

Fig. 2 Number of years of annual treatment to achieve elimination of transmission of S. mansoni as a function of coverage of SAC versus adults for low-transmission settings and low to high worm burden of infection in adults in low-transmission settings (baseline =9\%). NA: not achievable within 15 years of annual MDA. Results shown are generated using the ICL model

adults) can achieve EOT for low and high adult burden of infection. The number of years of annual treatment depends on the coverage level for both SAC and adults and the worm burden in adults. Using intensive treatment strategies (100\% of SAC and $100 \%$ of adults) can achieve EOT within 5 and 6 years for low and high adult burden of infection, respectively. Less intensive strategies, such as treating at least $70 \%$ of SAC and $50 \%$ of adults can achieve the EOT within 10 years of annual treatment if the burden of infection in adults is low. For a high burden of infection in adults, this target can be met within the same time, by treating $70 \%$ of SAC and $70 \%$ of adults.

\section{Discussion}

The 2030 goal for schistosomiasis is EPHP, to be achieved in 78 countries (Table 1), and the end goal is interruption of transmission, achieved when the incidence of infection reduces to zero, to be achieved by 2030 in 25 countries. In this study, we explore various treatment strategies and assess whether it is possible to eliminate schistosomiasis by using MDA alone, and if so, what treatment coverage is required for $\mathrm{SAC}$ and adults.

We find that the outcome depends on the transmission setting (low and high $\mathrm{R}_{0}$ values), worm burden in adults, MDA coverage level and the length of the annual treatment MDA programme. For low-transmission settings with a low adult burden of infection, treating SAC only cannot achieve the EOT goal (Table 3). However, we can achieve this goal within 9 years if we initially treat $75 \%$ of SAC annually for up to 3 years (time to EPHP) and then treat $75 \%$ of SAC plus $30 \%$ of adults for the next 6 years. Using more intensive strategies such as treating $90 \%$ of
SAC and $80 \%$ of adults annually can achieve EOT within 6 years of the MDA programme (Fig. 2).

For low-transmission settings with a high adult burden of infection (Table 4), like results for low adult burden of infection, we cannot achieve EOT by treating SAC only. However, we can achieve this goal within 13 years if we initially treat $75 \%$ of SAC annually for up to 4 years (time to EPHP) and then treat $75 \%$ of SAC plus $30 \%$ of adults for the next 9 years. Again, more intensive strategies can achieve this goal within a shorter time frame (Fig. 2). It should be noted here that results for low-transmission settings are generated using the ICL model only, as the SCHISTOX model typically cannot reproduce endemic prevalence less than about $12 \%$.

In moderate-transmission settings with a low adult burden of infection, treating $75 \%$ of SAC and $40 \%$ of adults annually, after the achievement of EPHP, can eliminate transmission within 9 years. For high adult burden of infection, analysis suggests that treating $75 \%$ of SAC and $55 \%$ of adults annually after the EPHP has been met can achieve the EOT within 10 years.

In high-transmission settings, regardless of the burden of infection in adults, there is a cut-off prevalence where more intensive strategies, such as higher coverage of SAC and adults, are required to eliminate transmission. This threshold varies with the age-profile of infection and across the two models used in this study.

It should be noted that, even when the EPHP goal has been achieved, higher coverage levels amongst SAC and adults are required to move towards EOT. Specifically, the coverage amongst SAC varies between 75 and 95\% and the coverage amongst adults varies between 30 and $75 \%$, depending on the baseline prevalence and the worm 
burden in adults. This is because, although the heavyintensity prevalence in SAC is reduced to less than $1 \%$ (for the EPHP), the prevalence in SAC and adults remains high. A large reduction in the prevalence of heavy-intensity infection does not necessarily correspond to a large reduction in the prevalence of infection. After the EPHP has been achieved, there might be light- to moderateintensity infections in SAC and light- to heavy-intensity infections in the non-SAC age categories. Therefore, stopping MDA after this goal has been achieved will lead to a resurgence in infection across all age classes [20].

Even though the EOT goal is achievable for all transmission settings and levels of infection in adults (with prolonged programmes), in some settings it might not be feasible to achieve high coverage levels, particularly amongst adults due to limited PZQ availability and hardto-reach adult individuals.

An important consideration is that preschool-age children (pre-SAC) have not been included in the treatment programme, due to the absence of clinical data on the drug safety and efficacy for young children [22]. A phase III study is underway to evaluate the efficacy and safety of a new PZQ formulation, developed by the Paediatric Praziquantel Consortium [23]. This new formulation is a small tablet with an acceptable taste for this age category. However, our results show that even when excluding preSAC from the treatment programme, transmission elimination can be achieved. Inclusion of this age group in MDA programmes can shorten the time to EOT as well as reduce the coverage level required in SAC and adults.

Consideration of the impact of animal reservoirs and understanding the importance of potential zoonotic species in transmission within human communities will become increasingly important as infection in human populations is reduced to low levels and nears potential elimination. The schistosomiasis transmission models will require extension to multi-host frameworks to quantify the risk of animal reservoirs sustaining transmission in human communities and to help inform what and where complementary 'One Health' interventions may be required [24, 25].

The prevalence of infection in our study is measured using a single Kato-Katz on two samples per individual [26]. However, for programmes aiming at EPHP or EOT, the Kato-Katz diagnostic technique suffers from low sensitivity to detect infection at very low prevalence [27]. Identifying low-prevalence endemic settings and tackling low-prevalence resurgent infection in post-treatment surveillance may require different diagnostic techniques. The point-of-care circulating cathodic antigen (POCCCA) is an alternative diagnostic technique which has greater sensitivity than Kato-Katz and performs better at detecting infection in low-prevalence endemic settings [28-30].

In this work, we have assumed random adherence to treatment with a fixed MDA coverage level. However, this may not always be the case, and as a result, our predictions may be on the optimistic side. Persistent noncompliers with MDA treatment can serve as reservoirs of infection. There is a clear need for more studies of compliance patterns in PZQ MDA-treated communities. We can address this issue by collecting and recording data on individual compliance, but to date very little attention has been paid to this during monitoring and evaluation of schistosomiasis control programmes.

Schistosomiasis can be highly heterogeneous on small scales, which in combination with human movement can impact the success of the MDA by reducing the probability of elimination [31]. As programmes transition towards EOT, understanding how observed heterogeneity in endemicity patterns influences and is influenced by transmission and control measures becomes crucial. Future analysis will be extended to integrate the current transmission model with models of human movement and to determine the optimal strategy for each spatial scale (district, subdistrict and community/village).

In this paper, we have not included the impact of acquired immunity, as it is not possible to distinguish the effects of immunity from the age-dependent exposure to infection. Both are probably important determinants of the observed patterns of infection across age classes. Some level of immunity is believed to slowly build up over long periods of exposure, which can lessen the impact of MDA in achieving elimination [32-34].

As MDA does not prevent reinfection, snail control can be an important component of the current schistosomiasis elimination strategy, especially in high-transmission areas. Previous modelling by $\mathrm{Li}$ and colleagues [43] showed that the addition of snail control can reduce the duration of treatment required to achieve elimination. While this treatment strategy can increase the impact, the lack of efficacy data limits model predictions of its potential benefits.

Much more attention in future modelling studies should be given to the impact of MDA plus snail control in reducing the prevalence of infection.

Additional treatment interventions include improving water, sanitation and hygiene (WASH), or vaccination if one becomes available in the coming years.

\section{Conclusions}

In conclusion, we find that treating SAC only cannot achieve EOT within a feasible time frame. However, it is possible to achieve this target if high and 
moderate-to-high annual treatment coverage is administered in SAC and adults.

\section{Acknowledgements}

We thank Jaspreet Toor for helpful discussions on the study.

\section{Authors' contributions}

$\mathrm{KK}, \mathrm{DA}, \mathrm{TDH}$ and RMA contributed to the design of the study. KK and DA ran the simulations, conducted the analyses and drafted the original manuscript KK, DA, TDH and RMA reviewed and edited the manuscript. All authors read and approved the final manuscript.

\section{Funding}

KK, DA, TDH and RMA gratefully acknowledge research grant support from the Bill and Melinda Gates Foundation (Grant No. OPP1184344) through the NTD Modelling Consortium.

\section{Availability of data and materials}

All data generated or analysed during this study are included in this published article.

\section{Declarations}

Ethics approval and consent to participate

Not applicable.

\section{Consent for publication}

Not applicable.

\section{Competing interests}

The authors declare that they have no competing interests.

\section{Author details}

${ }^{1}$ London Centre for Neglected Tropical Disease Research, London, UK. ${ }^{2}$ Department of Infectious Disease Epidemiology, School of Public Health, Faculty of Medicine, St Mary's Campus, Imperial College London, London, UK. ${ }^{3}$ MRC Centre for Global Infectious Disease Analysis, London, UK. ${ }^{4}$ Big Data Institute, Li Ka Shing Centre for Health Information and Discovery, University of Oxford, Oxford OX3 7LF, UK. ${ }^{5}$ The DeWorm3 Project, The Natural History Museum of London, London, UK.

Received: 16 July 2021 Accepted: 25 January 2022

Published online: 14 February 2022

\section{References}

1. Anderson RM, Turner HC, Farrell SH, Truscott JE. Studies of the transmission dynamics, mathematical model development and the control of schistosome parasites by mass drug administration in human communities. Adv Parasitol. 2016:94:199-246.

2. WHO. Schistosomiasis. https://www.who.int/news-room/fact-sheets/ detail/schistosomiasis. Accessed 24 Jun 2021

3. Kura K, Hardwick RJ, Truscott JE, Toor J, Hollingsworth TD, Anderson RM. The impact of mass drug administration on Schistosoma haematobium infection: what is required to achieve morbidity control and elimination? Parasite Vectors. 2020;13(1):554.

4. Zhang W, Molehin AJ, Rojo JU, Sudduth J, Ganapathy PK, Kim E, et al. Sm-p80-based schistosomiasis vaccine: double-blind preclinical trial in baboons demonstrates comprehensive prophylactic and parasite transmission-blocking efficacy. Ann N Y Acad Sci. 2018;1425:38-51.

5. Zhang W, Ahmad G, Le L, Rojo JU, Karmakar S, Tillery KA, et al. Longevity of Sm-p80-specific antibody responses following vaccination with $\mathrm{Sm}$-p80 vaccine in mice and baboons and transplacental transfer of Sm-p80-specific antibodies in a baboon. Parasitol Res. 2014;113:2239-50.

6. Kura K, Truscott JE, Toor J, Anderson RM. Modelling the impact of a Schistosoma mansoni vaccine and mass drug administration to achieve morbidity control and transmission elimination. PLoS Negl Trop Dis. 2019:13:e0007349.
7. Kura K, Collyer BS, Toor J, Truscott JE, Hollingsworth TD, Keeling MJ, et al. Policy implications of the potential use of a novel vaccine to prevent infection with Schistosoma mansoni with or without mass drug administration. Vaccine. 2020;38:4379-86.

8. WHO. Ending the neglect to attain the Sustainable Development Goals: A road map for neglected tropical diseases 2021-2030

9. WHO. Schistosomiasis: progress report 2001-2011, strategic plan 2012-2020. WHO. 2017

10. WHO. Helminth control in school-age children. A guide for managers of control programmes. Geneva World 2011.

11. 5th progress report: Reaching a Billion | Uniting to Combat NTDs. https:// unitingtocombatntds.org/reports/5th-report/. Accessed 24 Jun 2021.

12. Kura K, Ayabina D, Toor J, Hollingsworth TD, Anderson RM. Disruptions to schistosomiasis programmes due to COVID-19: An analysis of potential impact and mitigation strategies. Trans R Soc Trop Med Hyg. 2021:115(3):236-44.

13. Ayabina D, Kura K, Toor J, Graham M, Anderson RM, Hollingsworth TD. Maintaining low prevalence of Schistosoma mansoni: modeling the effect of less frequent treatment. Clin Infect Dis. 2021;72(Suppl 3):S140-5.

14. Toor J, Rollinson D, Turner HC, Gouvras A, King CH, Medley GF, et al. Achieving elimination as a public health problem for Schistosoma mansoni and S. haematobium: when is community-wide treatment required? J Infect Dis. 2019;221(Suppl 5):S525-30.

15. Anderson RM, May RM. Helminth infections of humans: mathematical models, population dynamics, and control. Adv Parasitol. 1985;24:1-101.

16. Anderson RM, May RM. Population dynamics of human helminth infections: Control by chemotherapy. Nature. 1982;297(5867):557-63.

17. Anderson RM, May RM. Infectious diseases of humans: dynamics and control. Oxford: Oxford University Press; 1991.

18. Truscott JE, Turner HC, Farrell SH, Anderson RM. Soil-transmitted helminths: mathematical models of transmission, the impact of mass drug administration and transmission elimination criteria. Adv Parasitol. 2016:94:133-98.

19. Graham M, Ayabina D, Lucas TC, Collyer BS, Medley GF, Hollingsworth TD, et al. SCHISTOX: An individual based model for the epidemiology and control of schistosomiasis. Infect Dis Model. 2021:6:438-47.

20. Toor J, Truscott JE, Werkman M, Turner HC, Phillips AE, King CH, et al. Determining post-treatment surveillance criteria for predicting the elimination of Schistosoma mansoni transmission. Parasit Vectors. 2019;12(1):437

21. Mekete K, Ower A, Dunn J, Sime H, Tadesse G, Abate E, et al. The Geshiyaro Project: A study protocol for developing a scalable model of interventions for moving towards the interruption of the transmission of soil-transmitted helminths and schistosome infections in the Wolaita zone of Ethiopia. Parasit Vectors. 2019;12:503.

22. Montresor A, Garba A. Treatment of preschool children for schistosomiasis. Lancet Glob Health. 2017:5(7):e640-1.

23. Pediatric Praziquantel Consortium. https://www.pediatricpraziquante Iconsortium.org/.

24. Léger E, Borlase A, Fall CB, Diouf ND, Diop SD, Yasenev L, et al. Prevalence and distribution of schistosomiasis in human, livestock, and snail populations in northern Senegal: a One Health epidemiological study of a multi-host system. Lancet Planet Heal. 2020:4:e330-42.

25. Borlase A, Webster JP, Rudge JW. Opportunities and challenges for modelling epidemiological and evolutionary dynamics in a multihost, multiparasite system: Zoonotic hybrid schistosomiasis in West Africa. Evol Appl. 2018;11:501-15.

26. Katz N, Chaves A, Pellegrino JP. A simple device for quantitative stool thick-smear in Schistosoma mansoni. Rev Inst Med Trop. 1999:14(6):397-400.

27. Kittur N, Castleman JD, Campbell CH, King CH, Colley DG. Comparison of Schistosoma mansoni prevalence and intensity of infection, as determined by the circulating cathodic antigen urine assay or by the Kato-Katz fecal assay: A systematic review. Am J Trop Med Hyg. 2016;94(3):605-10.

28. Lamberton PHL, Kabatereine NB, Oguttu DW, Fenwick A, Webster JP. Sensitivity and Specificity of Multiple Kato-Katz Thick Smears and a Circulating Cathodic Antigen Test for Schistosoma mansoni Diagnosis Pre- and Post-repeated-Praziquantel Treatment. PLoS Negl Trop Dis. 2014;8(9):e3139.

29. Prada JM, Touloupou P, Adriko M, Tukahebwa EM, Lamberton PHL, Hollingsworth TD. Understanding the relationship between egg- and 
antigen-based diagnostics of Schistosoma mansoni infection pre- and post-treatment in Uganda. Parasit Vectors. 2018;11(1):21.

30. Bärenbold O, Garba A, Colley DG, Fleming FM, Haggag AA, Ramzy RMR, et al. Translating preventive chemotherapy prevalence thresholds for Schistosoma mansoni from the Kato-Katz technique into the point-ofcare circulating cathodic antigen diagnostic test. PLoS Negl Trop Dis. 2018;2(12):e0006941.

31. Vegvari C, Truscott JE, Kura K, Anderson RM. Human population movement can impede the elimination of soil-transmitted helminth transmission in regions with heterogeneity in mass drug administration coverage and transmission potential between villages: A metapopulation analysis. ParasiteVectors. 2019;12(1):438.

32. Anderson RM, May RM. Herd immunity to helminth infection and implications for parasite control. Nature. 1985;315(6019):493-6.

33. Woolhouse MEJ, Taylor P, Matanhire D, Chandiwana SK. Acquired immunity and epidemiology of Schistosoma haematobium. Nature. 1991;351(6329):757-9.

34. Mitchell KM, Mutapi F, Mduluza T, Midzi N, Savill NJ, Woolhouse MEJ Predicted impact of mass drug administration on the development of protective immunity against Schistosoma haematobium. PLoS Negl Trop Dis. 2014;8:e3059.

35. Control of Neglected Tropical Diseases. https://www.who.int/teams/contr ol-of-neglected-tropical-diseases/preventive-chemotherapy/pct-datab ank/schistosomiasis.

36. De Vlas SJ, Gryseels B, Van Oortmarssen GJ, Polderman AM, Habbema JD. A model for variations in single and repeated egg counts in Schistosoma mansoni infections. Parasitology. 1992;104(Pt 3):451-60.

37. de Vlas SJ, Nagelkerke NJ, Habbema JD, van Oortmarssen GJ. Statistical models for estimating prevalence and incidence of parasitic diseases. Stat Methods Med Res. 1993;2(1):3-21.

38. Truscott JE, Gurarie D, Alsallaq R, Toor J, Yoon N, Farrell SH, et al. A comparison of two mathematical models of the impact of mass drug administration on the transmission and control of schistosomiasis. Epidemics. 2017;18:29-37.

39. Turner HC, Truscott JE, Bettis AA, Farrell SH, Deol AK, Whitton JM, et al. Evaluating the variation in the projected benefit of community-wide mass treatment for schistosomiasis: Implications for future economic evaluations. Parasit Vectors. 2017;10(1):213.

40. Fulford AJ, Butterworth AE, Ouma JH, Sturrock RF. A statistical approach to schistosome population dynamics and estimation of the life-span of Schistosoma mansoni in man. Parasitology. 1995;110(Pt 3):307-16.

41. Zwang J, Olliaro PL. Clinical efficacy and tolerability of praziquantel for intestinal and urinary schistosomiasis - a meta-analysis of comparative and non-comparative clinical trials. PLoS NegI Trop Dis. 2014;8:e3286.

42. WHO Expert Committee on the control of schistosomiasis. Prevention and control of schistosomiasis and soil-transmitted helminthiasis: report of a WHO expert committee. WHO 2002.

43. Li EY, Gurarie D, LO NC, Zhu X, King CH. Improving public health control of schistosomiasis with a modified WHO strategy: a model-based comparison study. Lancet Glob Health. 2019;7(10):e1414-22.

\section{Publisher's Note}

Springer Nature remains neutral with regard to jurisdictional claims in published maps and institutional affiliations.

Ready to submit your research? Choose BMC and benefit from:

- fast, convenient online submission

- thorough peer review by experienced researchers in your field

- rapid publication on acceptance

- support for research data, including large and complex data types

- gold Open Access which fosters wider collaboration and increased citations

- maximum visibility for your research: over $100 \mathrm{M}$ website views per year

At BMC, research is always in progress.

Learn more biomedcentral.com/submissions 